\title{
The Design and Development of Flexsim/JMP based Quality Simulation System for Mass Customization
}

\author{
Chen Si-Yu ${ }^{12, a}$, Fan Shu-Haii ${ }^{* 2, ~ b}$, Xiong Jia-Wei ${ }^{12, c}$, Zhang Wen-Qian ${ }^{12, d}$ \\ ${ }^{1}$ Department of Industrial Engineering, Nanjing Tech University, Nanjing 210009, China \\ ${ }^{2}$ MIT Quality Information Program “Data Quality \& Info Security” Lab, Cambridge, MA, USA

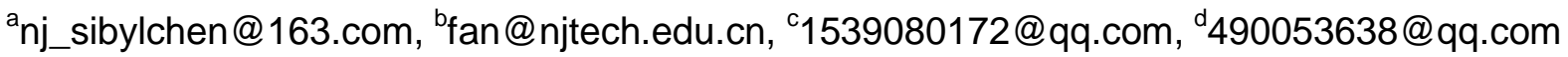

\begin{abstract}
Keywords: Mass Customization; JMP; Quality Management and Control; Flexsim; Openness Research

Abstract. As the latest quality control application, JMP has more advantages than Minitab, which is commonly used recent years, for it has better capability of dealing with and mining data, as well as its good automation ability. Therefore, it can be well used to analysis on large batch of data. At the other hand, simulation software Flexsim obtain valid quality information of the products by modeling the production process, in order to complete prior prevent and mass customization manufacture quality control effectively. So, this thesis focuses on the design of open interaction system based on the good automation ability of JMP through both static and dynamic method to improve the efficiency of the existing quality control system.
\end{abstract}

\section{Introduction}

Mass Customization refers to provide customization products in any quantity for single customer or market with special demand, while its cost and production rate are the same as mass proction[1]. It can be said that it is the derivative and improvement of the traditional mass production mode under the demand of market. Mass customization manufacture aims to reduce the waste of money cost cuased by production diversification and the inventory of enterprise, to obtain greater profit space, so as to improve the competitiveness of the enterprises. Mass customization production quality control system is based on continuous improvement in production, which emphasis on pre-prevention and comprehensive quality management, pay attention to self-test among the module, and make use of statistical process control for productions large in quatities, small in batch at proper time[2,3,4]. Nowadays, Multivariate quality control is a major means of mass customization production quality control, Quality simulation and quality statistical process control are respectively the main quality control methods during manufacturing and inspection periods. In system simulation aspect, object-oriented visualization $3 \mathrm{~d}$ modeling and simulation software, Flexsim, can obtain quality data by modeling production process, while it also has good ability of second development to interact with other tools for data exchange[5]. In terms of statistics, as the latest cross-platform statistical tool, JMP has more powerful data processing ability, and better scripting automation ability than other statistical tools, so it can have better performance in completing data statistics of production[6]. However, it can't meet the requirements of the mass customization manufacturing quality control effectively when these two tools operating separately. So, there is a need for an open interaction between these two tools: simulating the process of mass customization by Flexsim, then share it with JMP in real-time for further analysis, so as to study the quality level of the whole production process rapidly and accurately. This thesis mainly discuss on the design and development of Flexsim/JMP based quality simulation system for mass customization.

\section{The design of Flexsim/JMP based quality simulation system}

The overall design scheme of the Flexsim/JMP based quality simulation system is shown as the below figure:Flexsim is responsible for simulating the production process and recording the data, 
then connect to underlying tool JMP for statistics through middleware(no need for static connection). This interaction scheme can be divided into two types: static and dynamic. Static connection directly export the data from Flexsim through Excel menu command, with no need for middleware, while JMP access to the table offline. The design of dynamic connection is more complex, which need to select proper interface of JMP and Flexsim by listing all the available interfaces of them.

Flexsim has three kinds of interactive ports: 1) ODBC(Open Database Connectivity), used to connect with database like Access, Oracle and so on; 2) DDE connection(Dynamic Data Exchange), can connect with any opened Excel table, and write on the table at the same time; 3 ) other methods, like using Files or Sockets or other commands to finish real-time connection.

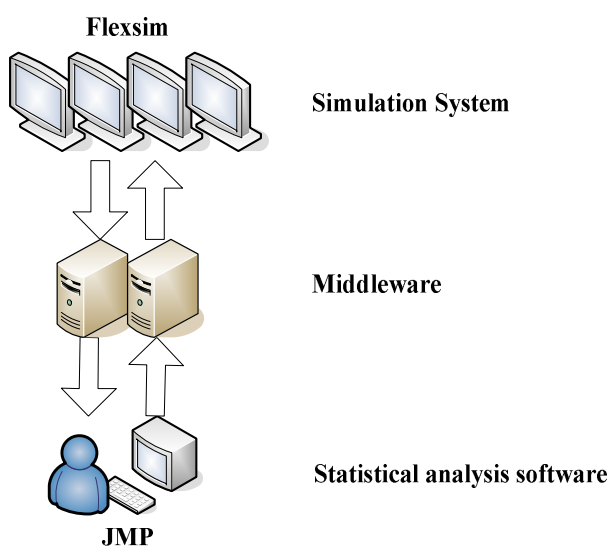

Fig.1 Overall design scheme of the system

The connection between JMP and external programs can be divided into static and dynamic. Dynamic connection can not only support data exchange with external programs through JSL script, but also can share data through other programming languages like VB, VC++. Details are shown as below table:

Table 1 The list of connection methods of JMP

\begin{tabular}{|c|c|c|c|}
\hline \multicolumn{2}{|c|}{ connection type } & object type & Description \\
\hline \multirow{6}{*}{ Static } & \multirow{4}{*}{$\begin{array}{l}\text { no need for ODBC } \\
\text { driver }\end{array}$} & Internet file & $\begin{array}{l}\text { connect with the internet, and show the } \\
\text { data in the forms of text, webpage or excel } \\
\text { table }\end{array}$ \\
\hline & & Text file(.txt) & directly visit the text files \\
\hline & & SPSS file & connect with any file created by SPSS \\
\hline & & Minitab file & $\begin{array}{l}\text { connect directly with any file created by } \\
\text { Minitab }\end{array}$ \\
\hline & \multirow{2}{*}{ need for ODBC driver } & $\begin{array}{l}\text { Database } \\
\text { file(.dbf, .ndx, .mdx })\end{array}$ & $\begin{array}{l}\text { need for a relevant } \mathrm{V} 3+\text { driver to } \\
\text { complete connection }\end{array}$ \\
\hline & & Access file(.mdb) & $\begin{array}{l}\text { need for a relevant } \mathrm{V} 3+\text { driver to } \\
\text { complete connection }\end{array}$ \\
\hline \multirow{5}{*}{ Dynamic } & \multirow{4}{*}{ through JSL } & Datafeed & $\begin{array}{l}\text { A real-time method to read data } \\
\text { continuously, such as from a laboratory } \\
\text { measurement device connected to a serial } \\
\text { port }\end{array}$ \\
\hline & & $\begin{array}{l}\text { Dynamic } \\
\text { Libraries(DLLs) }\end{array}$ & $\begin{array}{l}\text { Extend JMP functionality by using JSL to } \\
\text { load a DLL and call functions exported by } \\
\text { that DLL. }\end{array}$ \\
\hline & & Sockets & $\begin{array}{l}\text { another method to establishing a live } \\
\text { datafeed, can be divided into stream } \\
\text { sockets and datagram sockets }\end{array}$ \\
\hline & & Excel table & $\begin{array}{l}\text { can be visited offline or script the } \\
\text { profiler interface to Excel }\end{array}$ \\
\hline & $\begin{array}{l}\text { through external } \\
\text { programming } \\
\text { languages }\end{array}$ & OLE Automation & $\begin{array}{l}\text { Most of JMP can be automated through } \\
\text { VB and VC++ with MFC }\end{array}$ \\
\hline
\end{tabular}


Considering the qualities of the interfaces of JMP and Flexsim, Excel is used as a middleware in this thesis for its great automation ability, accepting real-time data from simulation system and share it with JMP.

\section{The development of Flexsim/JMP based quality simulation system}

\section{The development of offline interaction system}

Flexsim can export the simulation result as an external Excel table through its menu command, while JMP can visit local database through ODBC function.

(1) Export simulation data tables from Flexsim:

Selecting statistics> statistics and report in menu of Flexsim, choose relevant data in the dialog, click on generate report and then data required is shown as an Excel table.

Otherwise, clicking on the Excel bottom directly in toolbar, and then selecting the required tables in the dialog.

(2) JMP visit local file through ODBC:

Clicking File> database> open table in JMP, choosing connect to connect to a database, then selecting Excel files in the list and broswering the computer to find the aimed files in the dialog. statement

In this dialog, worksheet can be specified and certain data can be exported through SQL

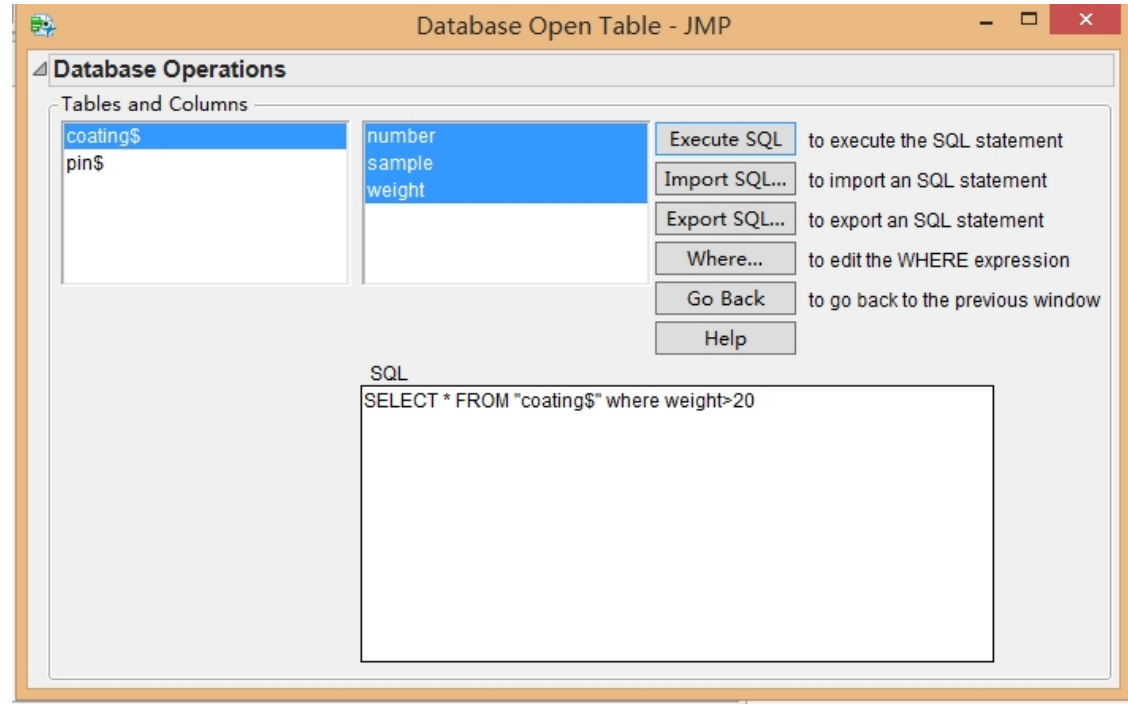

Fig. 2 Open table from database

\section{The development of online interaction system}

The dynamic interaction connection mainly automates JMP through VB language, and then completes real-time data sharing in Excel. Simulation tool Flexsim in the top-level can realize data exchange with Excel through DDE, detailed commands are shown as below[7]:

Excellaunch ( [ strlaunchdir]) //open Excel program, eg. Excellaunch ( "C:\C:IProgram Files (x86)LMicrosoft OfficelOffice12”)

Excelopen (str workbook) //open Excel file, eg. Excelopen ( "quality.xlsx")

Excelsetsheet (strsheetname) //specify worksheet, eg. Excelsetsheet ("sheet1")

Excelclose( num save[1/0]) //close worksheet in Excel file, eg. Excelclose (true)

Excelquit() //shut down Excel

After excuting these commands, the DDE connnection between Flexsim and Excel is completed, and the simulation data will be send to Excel table.

The dynamic interation of Excel and JMP is done through VBA in Excel itself. Selecting Development Tool>Visual Basic> Tool> Reference, then finding JMP and revelant choices in the dialog and ticking on these references like below. 


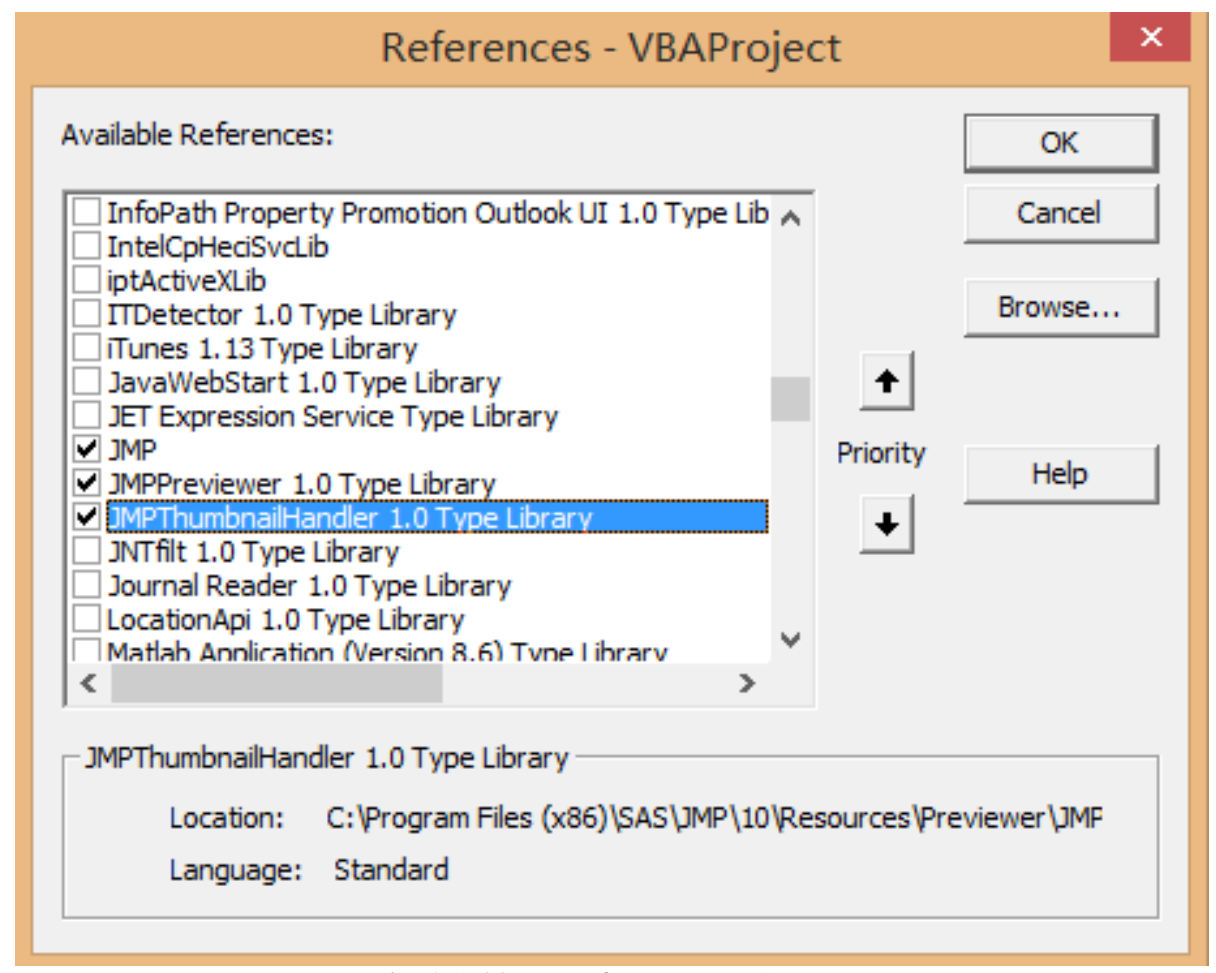

Fig. 3 Add DLL of JMP to VBA

Define a global variable at first, cilck on VBA Project> insert> module, input thses codes:

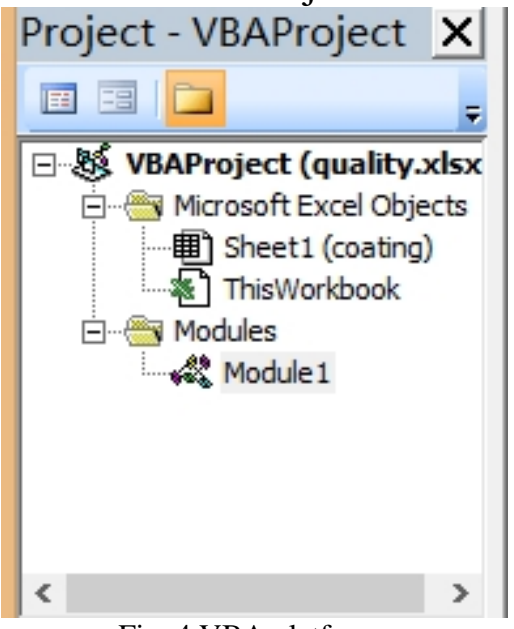

Fig. 4 VBA platform

Public MyJMP as JMP.Application // define JMP application

Public DT as JMP.DataTable // define JMP datatable

Public DocOpen as Boolean // remark JMP table whether is opened

In this interaction, there are some tips needed to be pay attention: when the change to Excel occurs on the firstline, JMP won't have any change; when the number of rows changed is beyond the number of JMP tracking now, JMP will automatically call AddRows function to create new lines[8].

According to the rules of JMP shown in below table, the main command for calling for function is like this:

Public Counter As Integer //count to make sure the data will be updated after every five changes

Public JMPDoc As JMP.Document //declare JMP document

Public CChart As JMP.ControlChart //declare JMP control chart

Public ChartOpen as Boolean //track on whether the gragh is opened

Public DB As AUTODB visible

Set MyJMP = CreateObject(“JMP.Application”) MyJMP.Visible=True //make JMP application 
Counter $=0$ //initialize variable counter

Private Sub Workbook_SheetChange(ByValSh As Object, ByVal Source As Range)

Counter $=$ Counter +1

If (Counter Mod $5=0$ Or Counter $=1)$ Then //send the update to the control charts in JMP after every 5 change

If Not (ChartOpen) Then

Set CChart = JMPDoc.CreateControlChart //create new control chart

CChart.LaunchAddProcess "Column 1" //add columns

CChart.LaunchAddSampleUnitSize 5 //set the size of sample unit

CChart.LaunchSetChartTypejmpControlChartVar//set the type of the charts

CChart.Launch //open the chart

ChartOpen $=$ True $/ /$ maek a chart opened

End If

Table 2 The description of subroutines of JMP

\begin{tabular}{|l|l|}
\hline subroutine(object name_event name) & Description \\
\hline Workbook_Open & $\begin{array}{l}\text { Be called when the Excel table is initially loaded. It } \\
\text { initializes some variables, starts JMP, and tells JMP } \\
\text { to open the same Excel file that is currently loaded } \\
\text { into Excel. }\end{array}$ \\
\hline Workbook_Change & $\begin{array}{l}\text { Be generated every time a user changes the data in } \\
\text { any cell in any worksheet in the workbook, it creates } \\
\text { a control chart in JMP using the current data table. }\end{array}$ \\
\hline Workbook_BeforeClose & $\begin{array}{l}\text { Be invoked when the Excel workbook is closed, but } \\
\text { before the window goes away. }\end{array}$ \\
\hline
\end{tabular}

\section{The application of Flexsim/JMP based quality simulation system}

This part mainly simulates the assembly line of mass customization through Flexsim, and then make real-time monitoring of the weight of the products, carry out the quality control on weight indicator.

Tag for the weight parameter of the product at first, for example:

Addlabel(obj object, strlabelname) // add tags to entities

Setlabelstr(obj object, str/num label, str value) // set the name of the label

Setlabelnum(obj object, str/num label, numvalue) //set the value of the label

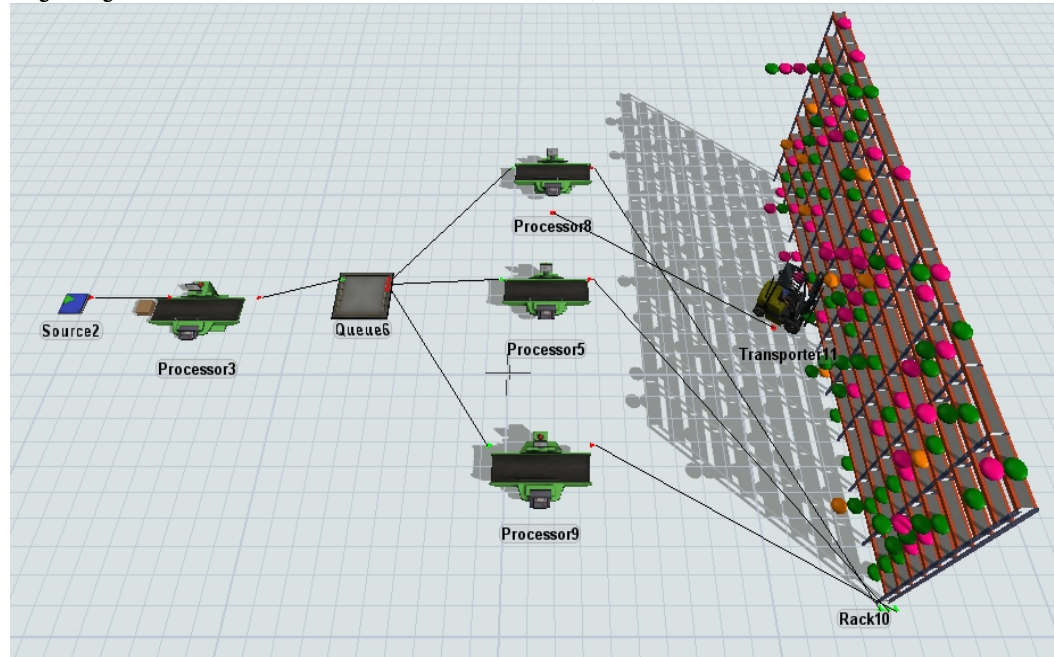

Fig. 5 Simulation of the operation of mss customization 
Making a dynamic connection between Flexsim and JMP using the method mentioned above, to make a control chart in JMP for the weight of the continuous 40 products produced by the simulation system. Set the size of the sample five, and then get the Xbar-R control charrt as below. Judge the weight indicator by observing the location of the points according to the eight criterias of control charts[9].(GB/T 4091-2001)

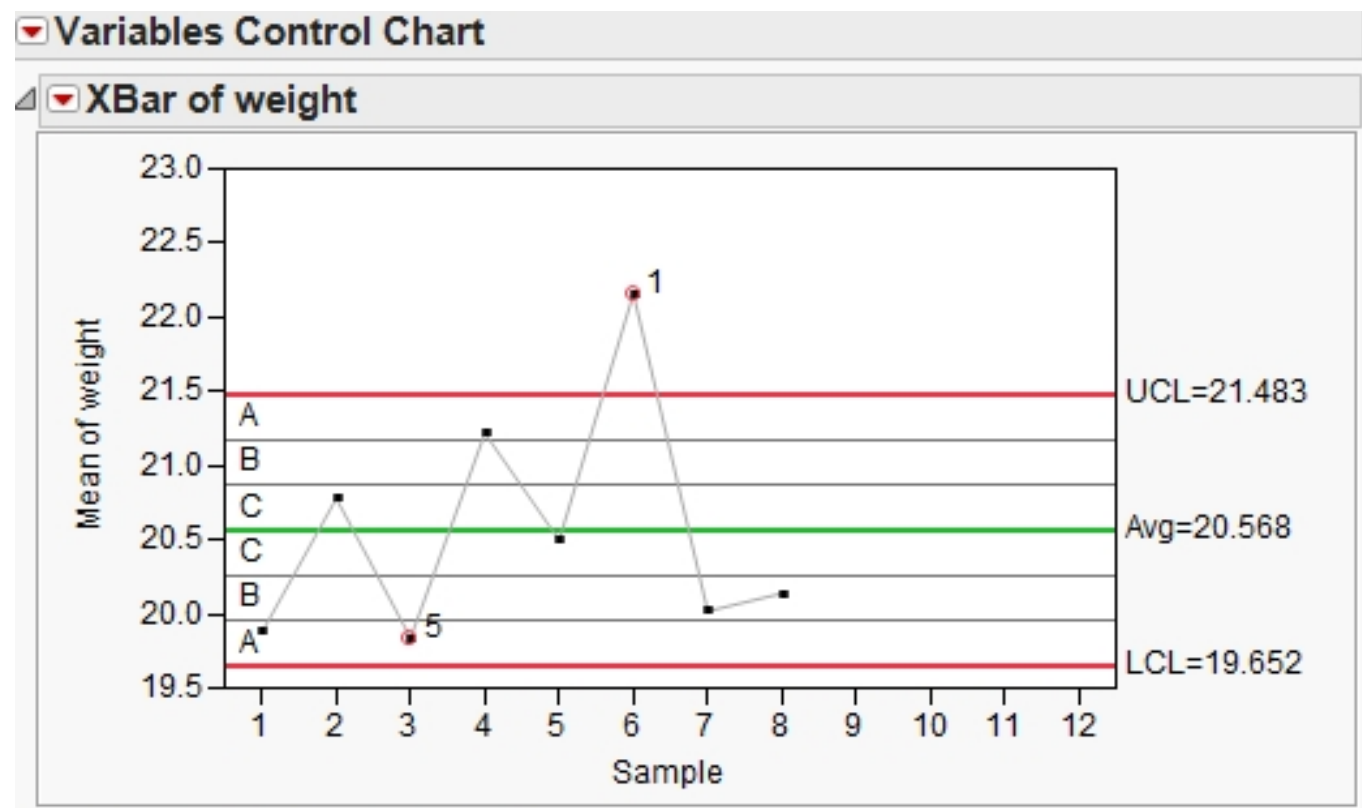

Note: The sigma was calculated using the range.

Fig. 6 Xbar-R control chart of the weight indicator(average)

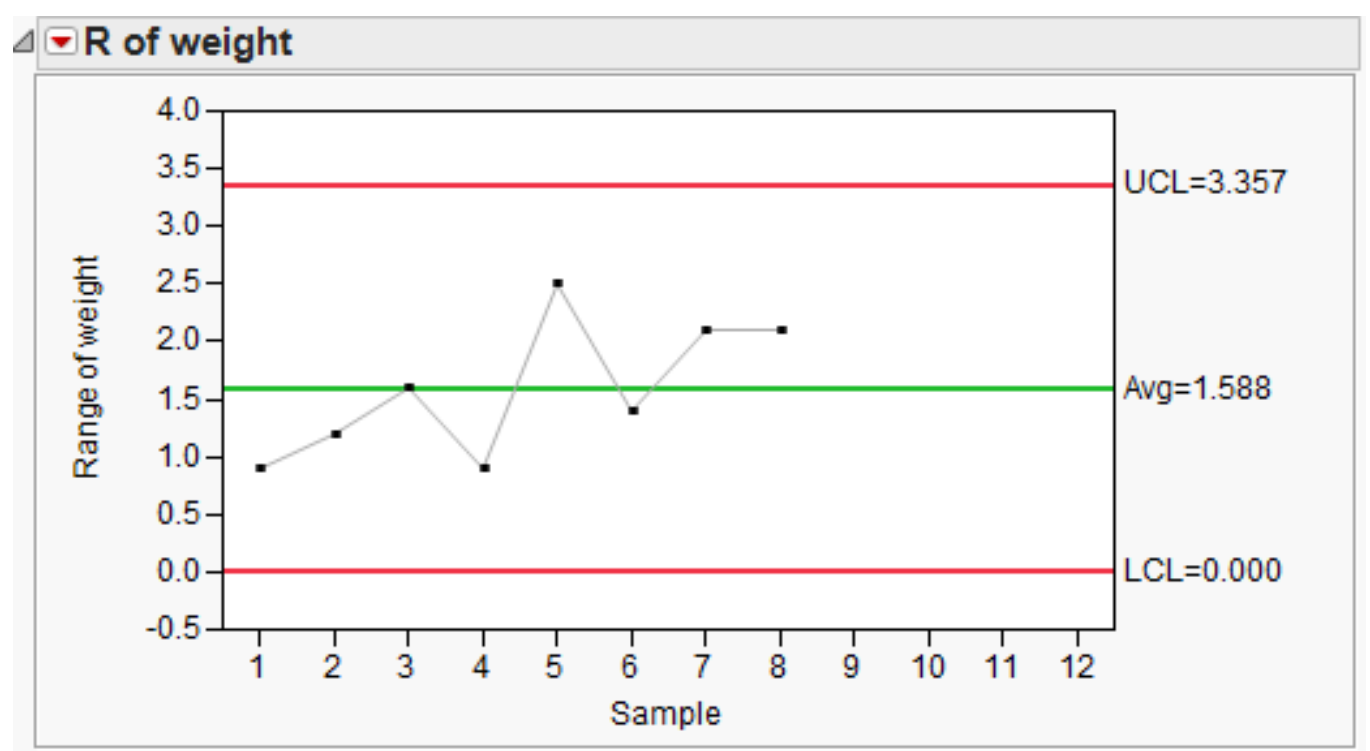

Fig. 7 Xar-R control chart of weight indicator(extremum)

Abnormal points have been showed on the figure 6, which conform to the first rule of the eight criterias "A point falls outside the area $\mathrm{A}$ " and the fifth rule "There are two points in three consecutively falls on the outside of B area on the same side of centerline". Since the process has been judged abnormal, six sigma management can be used to analyze and make improvement on the phenomenon and then restart the monitor of the data of the production simulation, so as to ensure the quality of the product. 


\section{Conclusion}

As a latest quality statistic control software, JMP can successfully realize real-time data sharing with database and system simulation tool with the help of its great ability of analysis and automation. So, it can conduct real-time comprehensive quality control on the production line simulated through the system to solve the problem of quality control for mass customization in a low cost and high efficiency way.

This thesis takes Flexsim as the example of simulation software, considering the characteristics of the external interfaces of both Flexsim and JMP, and then designs a Flexsim/JMP based quality simulation system to realize real-time data sharing between these two applications, which can be applicated into mass customization. This system takes fully advantage of both Flexsim on simulation and JMP on statistic, and provides the manufacture enterprise a solution to deal with the problem of quality control.

\section{Acknowledgements}

This work was supported by National Natural Science Foundation of China (Grant No.71671089), National Natural Science Foundation of China (Grant No.71171110) and National Natural Science Foundation of China (Grant No. 71371097)

\section{References}

[1] Lan-Juan Dong, Shu-Hai Fan and Ting-Hua Huang: Summary on the mass customization multivariate quality control. Modern Manufacturing Engineering Vol. 12 (2013), p. 125-129.

[2] Shu-Hai Fan, Tian-Yuan Xiao and Meng Hao: Study on Quality Assurance Technologies for Mass Customization. Aviation precision manufacturing technology Vol. 6 (2002), p. 1-3.

[3] Ming-Zhen Wen, Li-Ming Zhao and Min Zhang: Quality Control for Mass Customization. Modular Machine Tool \& Automatic Manufacturing Technique Vol. 2 (2006), p. 110-112.

[4] Shu-Hai Fan, Tian-Yuan Xiao, Pei-Huang Lou and Lin-Xuan Zhang: Model Building of the Dynamic Network in MCMQA. Journal of System Simulation Vol. 12 (2004), p. 2767-2773.

[5] Zhi Zhou, Shu-Hai Fan and Xia Cao: Flexsim based unified simulation platform of quality assurance. Manufacturing Automation Vol. 31 (2009), p. 34-36.

[6] Xiang Lei, Ling Jiang, Shao-Hua Zhang and Run-Ze Zhang: Research On The Secondary Development Methods Of JMP. Software Vol. 2 (2014), p. 48-49.

[7] Ting-Hua Huang, Shu-Hai Fan, Sai-Sai Duan and Lan-Juan Dong: The design and development of mass customization quality simulation system based on Flexsim/Minitab. Modern Manufacturing Engineering Vol. 2 (2014), p. 6-10.

[8] Information on http://www.jmp.com

[9] Ting-Hua Huang, Lan-Juan Dong, Tian Xu, Shu-Hia Fan and Ye-Xiang Fang: The Design of Minitab/DDE Based Six Sigma Management Integrated System and its Application in SMED. Modular Machine Tool \& Automatic Manufacturing Technique, Vol. 11 (2014), p. 156-160. 